\title{
Photosynthetic performance and growth responses of Liriope muscari (Decne.) L.H. Bailey (Asparagaceae) planted within poplar forests having different canopy densities
}

\section{CURRENT STATUS: UNDER REVISION}

BMC Ecology BMC Series

jiaojiao zhang

Nanjing Forestry Unversity

ORCiD: https://orcid.org/0000-0002-8716-2197

Jian Zhou

zhiwu@njfu.edu.cnCorresponding Author

Ling Zhu

Nanjing Forestry University

Xu Zhang

Nanjing Forestry University

DOI:

10.21203/rs.2.18505/v1

\section{SUBJECT AREAS}

Agroecology Ecological Modeling

\section{KEYWORDS}

chlorophyll fluorescence, diurnal course, light acclimation, light response curve, soil characteristics, path analysis 
Abstract

Background: Liriope muscari (Decne.) L.H. Bailey is a valuable horticultural and medicinal plant that grows under a range of light intensities, from high to low, in the understories of shrubs. To understand how this species adapts to these various environments, we selected two groups of lilyturf growing under poplar trees at two different spacings. Each group was divided into three types, open field, forest edge and shaded forest with high, medium and low irradiance levels, respectively, and then we examined their photosynthetic characteristics, physiology and biomasses.

Results: Light saturation point, light compensation point and in situ net photosynthetic rate (PN) were highest in lilyturf growing under high. In contrast, lilyturf growing under low light had a higher apparent quantum yield and $\mathrm{Chl}$ a and b contents, indicating that they adapted to low light. Although the leaves of lilyturf growing under low light were small, their root tubers were heavier.

Conclusions: The novelty of this research is the demonstration of the eco-physiological basis of lilyturf's shade adaptation mechanisms indicated by photosynthetic activity, chlorophyll fuorescence, Chla, Chlb and Car contents and grown in different irradiance. We believe that lilyturf is a shadetolerant plant suitable for planting in undergrowth, but attention should be paid to the canopy density of the forest when interplanting. The findings presented here advance our understanding of the photosynthetic characteristics of understory plants and may assist in the optimization of irradiances in the furture. Keywords: chlorophyll fluorescence; diurnal course; light acclimation; light response curve; soil characteristics; path analysis.

Full Text

Due to technical limitations, full-text HTML conversion of this manuscript could not be completed. However, the manuscript can be downloaded and accessed as a PDF.

Figures 


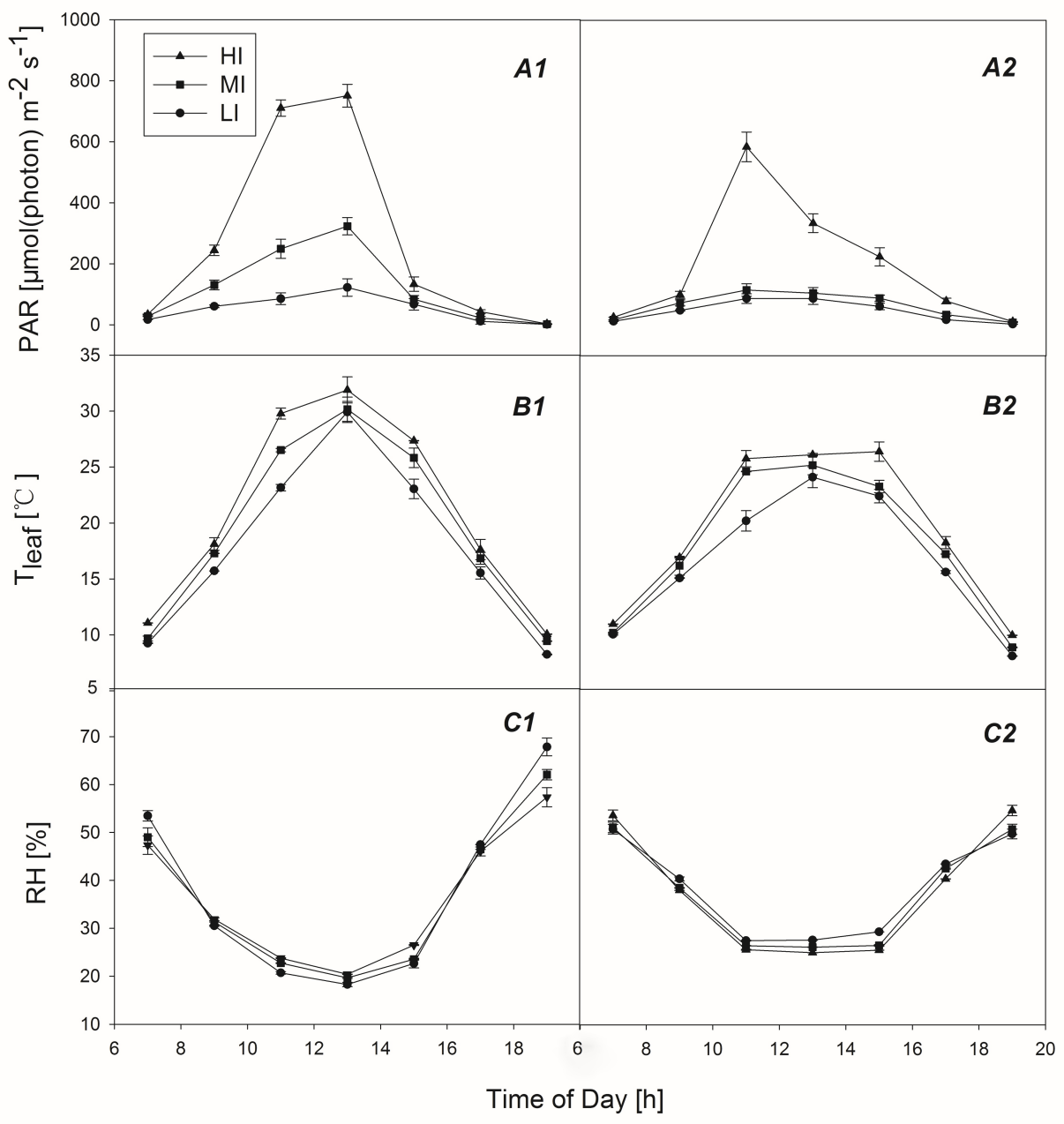

Figure 1

Diurnal changes in (A1-2) photosynthetically active radiation (PAR), (B1-2) leaf temperature (Tleaf), and (C1-2) relative humidity $(\mathrm{RH})$ inside the cuvette under different shade treatments in group1 $(A 1, B 1, C 1)$ and group2 $(A 2, B 2, C 2)$. Means $\pm S E(n=3)$. 


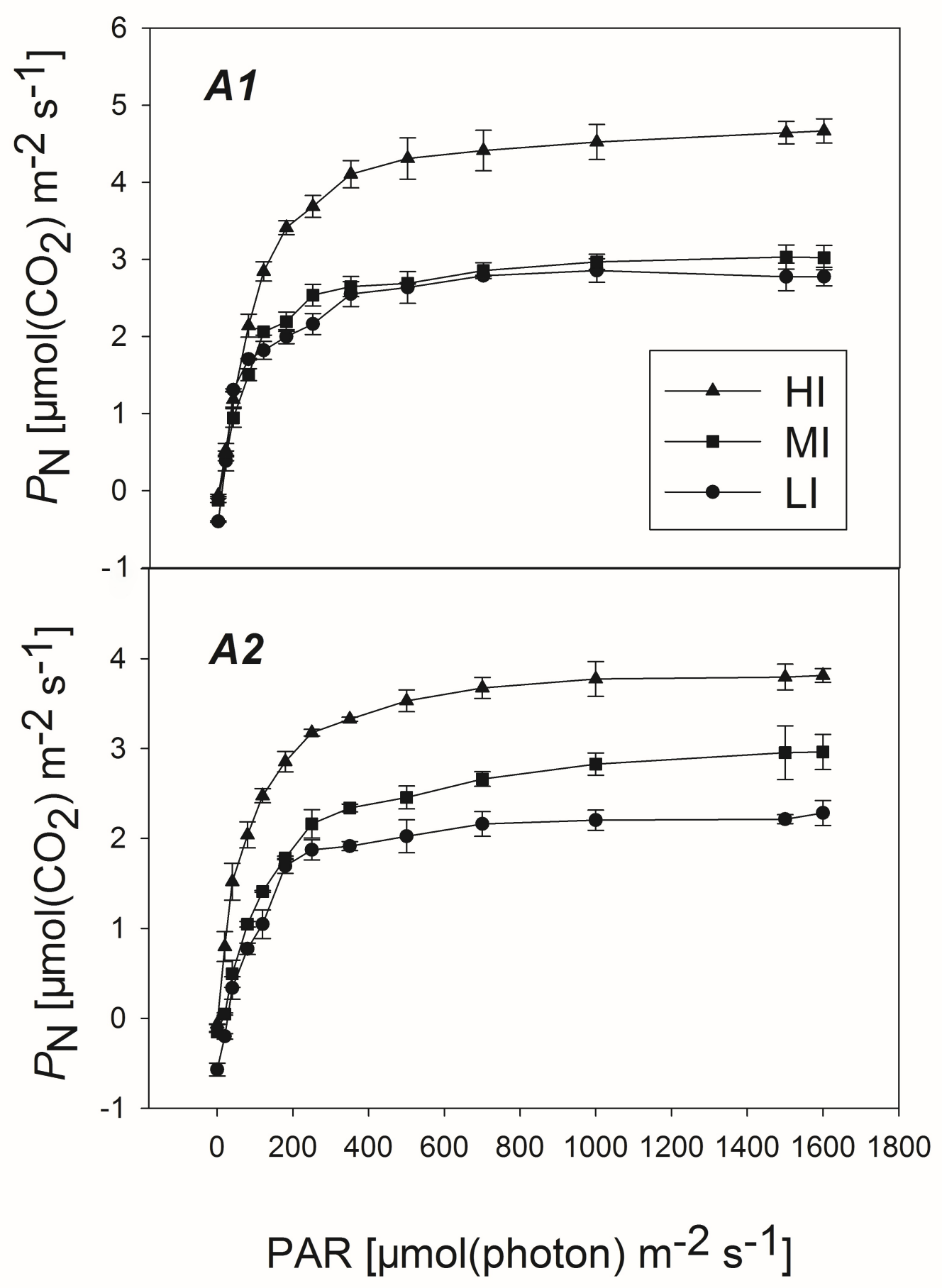

Figure 2

Response of net photosynthetic rate (PN) to PAR in Liriope muscari grown under high (HI), medium (MI) and low (LI) incident PAR in group1 (A1) and group2 (A2). Means \pm SE $(n=3)$.

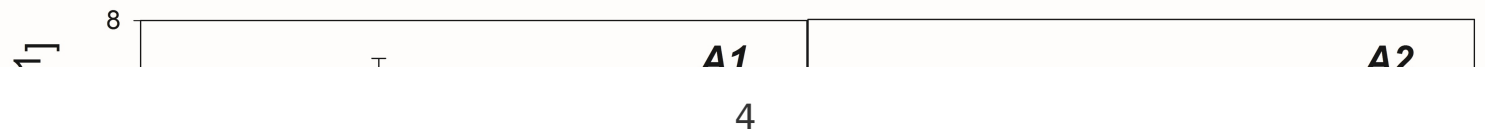




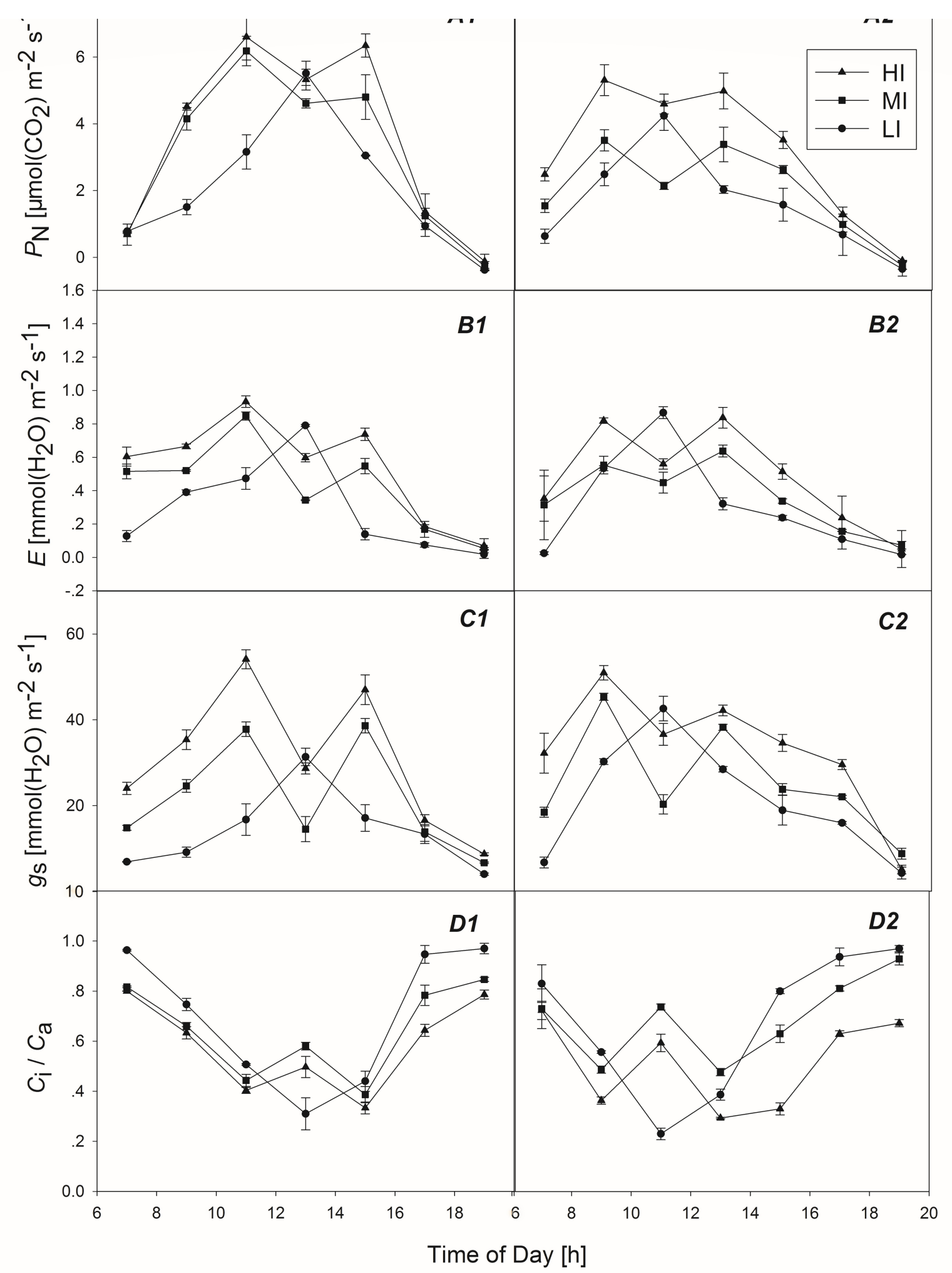

Figure 3

Changes in (A1-2) net photosynthetic rate (PN), (B1-2) transpiration rate (E), (C1-2) stomatal conductance (gs), and (D1-2) internal to ambient $\mathrm{CO} 2$ concentration (Ci/Ca) of Liriope muscari plants grown under high (HI), medium (MI) and low (LI) incident PAR in 
group1(A1,B1,C1,D1) and group2 $(A 2, B 2, C 2, D 2)$. Means $\pm S E(n=3)$.

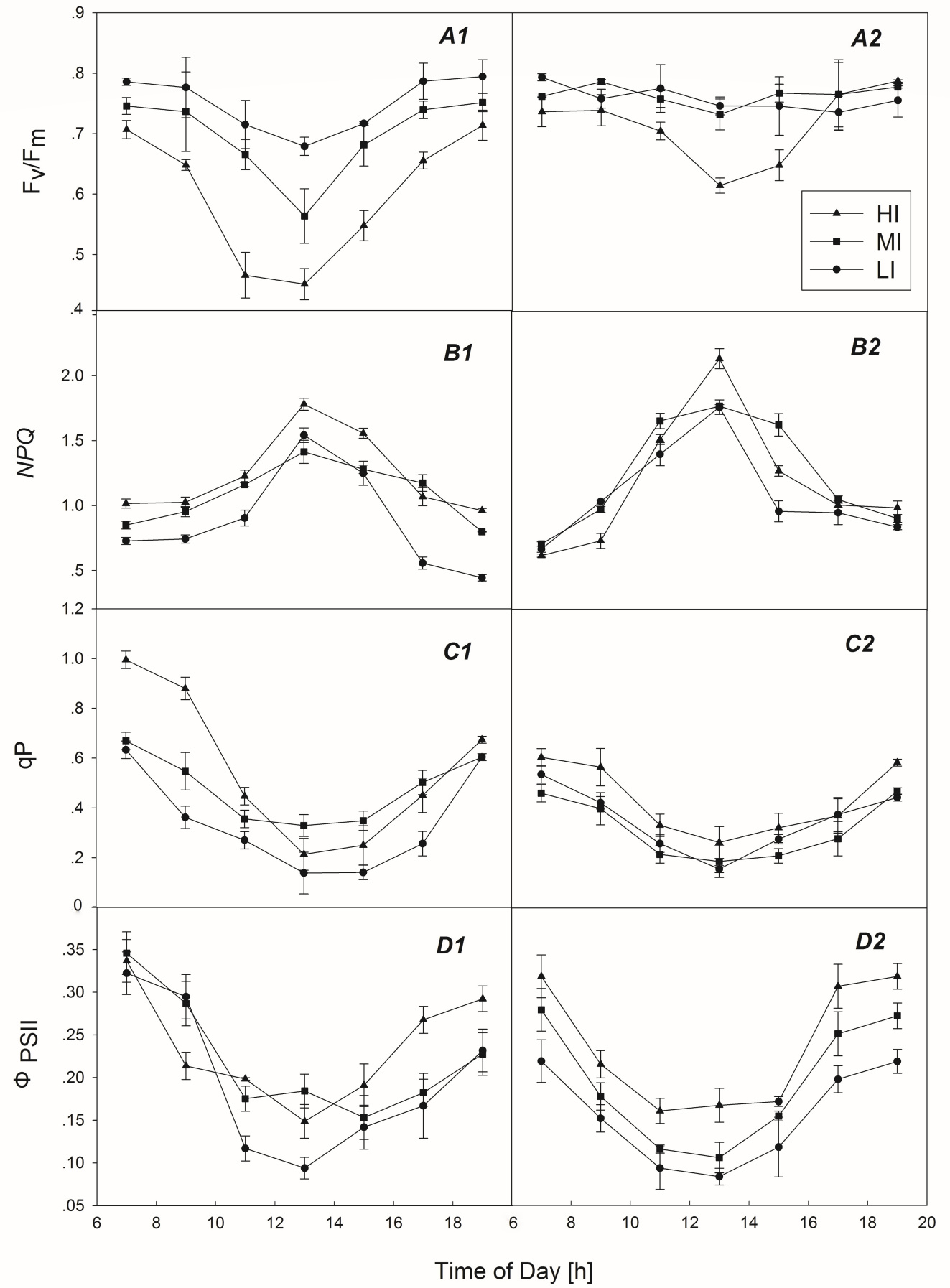

Figure 4

Diurnal changes in (A1-2) maximal photochemical efficiency (Fv/Fm), (B1-2) 
nonphotochemical quenching (NPQ), (C1-2) photochemical quenching (qP), and (D1-2)

quantum yield of PSII (ФPSII) of Liriope muscari plants grown under high (HI), medium (MI)

and low (LI) incident PAR in group1 $(A 1, B 1, C 1, D 1)$ and group2 $(A 2, B 2, C 2, D 2)$. Means \pm SE ( $n$ $=3$ ). 